"This is the peer-reviewed version of the following article: Angew. Chem. Int. Ed. 2014, 53, 13794 13797, which has been published in final form at DOI: 10.1002/anie.201408172. This article may be used for non-commercial purposes in accordance with Wiley-VCH Terms and Conditions for SelfArchiving."

\title{
Formation of dianions in helium nanodroplets
}

Andreas Mauracher, ${ }^{1}$ Matthias Daxner, ${ }^{1}$ Andreas Mauracher, ${ }^{1}$ Stefan E. Huber, ${ }^{1}$ Johannes Postler, ${ }^{1}$ Michael Renzler, ${ }^{1}$ Stephan Denifl, ${ }^{1}$ Paul Scheier ${ }^{1, *}$ and Andrew M. Ellis, ${ }^{2, *}$

1 Institut für Ionenphysik und Angewandte Physik, Universität Innsbruck, Technikerstr. 25, A-6020 Innsbruck, Austria

${ }^{2}$ Department of Chemistry, University of Leicester, University Road, Leicester, LE1 7RH, UK

Email: Paul.Scheier@uibk.ac.at; andrew.ellis@le.ac.uk

Abstract: The formation of dianions in helium nanodroplets is reported for the first time. The fullerene cluster dianions $\left(C_{60}\right)_{n}{ }^{2-}$ and $\left(C_{70}\right)_{n}{ }^{2-}$ were observed by mass spectrometry for $n \geq 5$ when helium droplets containing the appropriate fullerene were subjected to electron impact at approximately $22 \mathrm{eV}$. A new mechanism for dianion formation is described, which involves a twoelectron transfer from the metastable $\mathrm{He}^{-}$ion. As well as the prospect of studying other dianions at low temperature using helium nanodroplets, this work opens up the possibility of a wider investigation of the chemistry of $\mathrm{He}^{-}$, a new electron-donating reagent. 
Multiply charged anions (MCAs) are common constituents of chemical systems. However, when stripped of a counterionand/or solvent the Coulomb repulsion between the two or more excess electrons usually makes the anion unstable with respect to processes such as electron autodetachment or fission into singly charged anions (Coulomb explosion).[1-3] For this reason the study of isolated MCAs in the gas phase has proved a major experimental challenge.Nevertheless, MCAs have been detected in the gas phase in the past few decades. The earliest examples were certain large organic dianions, derived from substituted polyaromatic hydrocarbons, which are favorable because the excess negative charge can be distributed over a substantial volume, thus minimizing electrostatic repulsion.[4] More recently a variety of specialized techniques have been employed to generate MCAs in the gas phase. For example, surface sputtering using highly energetic cations as projectiles has yielded $C_{n}{ }^{2-}$ ions, where $n \geq 7,[5]$ whereas laser desorption has been used to form the fullerene dianions $\mathrm{C}_{60}{ }^{2-}$ and $\mathrm{C}_{70}{ }^{2-} .[6,7]$ Other important developments include the production of dianions and trianions of large metal clusters, including those of silver, gold, and lead.[8-10] These anions were made by using laser ablation to generate monoanions, which were then isolated in a Penning trap and flooded with low-energy electrons to make MCAs. Electrospray ionization is also a source of solvent-free MCAs, and examples studied include the carbon cluster dianions $\mathrm{C}_{84}{ }^{2-}$ and $\mathrm{C}_{90}{ }^{2-}$,[11] the citrate dianions,[12] and a variety of dicarboxylate dianions of formula $\left[\mathrm{OC}\left(\mathrm{CH}_{2}\right)_{n} \mathrm{CO}\right]^{2-}$.[13]

Herein we report the first observation of dianions produced in liquid helium nanodroplets. The focus of the current study is on dianions of $\mathrm{C}_{60}$ and $\mathrm{C}_{70}$ clusters. Aside from this original observation, there are two important underlying aspects of this work. First, we demonstrate a new method for producing and studying dianions, which may be applicable to MCAs beyond those covered in this paper. Second, evidence is presented for a new and unusual mechanism, which accounts for the formation of dianions in helium nanodroplets.

Figure 1a shows a negative-ion mass spectrum obtained at an electron energy of $22 \mathrm{eV}$ for helium droplets doped with $\mathrm{C}_{60}$. The dominant peaks in this mass spectrum arise from the monoanions $\left(\mathrm{C}_{60}\right)_{n}{ }^{-}$, which form a clear series. The production of monoanions in the gas phase from electron attachment to doped helium nanodroplets is already well known.[14, 15] Much weaker are additional peaks in the spectrum (Figure 1a) between $\mathrm{m} / \mathrm{z} 2000$ and 6000, which are precisely midway between the intense $\left(\mathrm{C}_{60}\right)_{n}{ }^{-}$peaks and are assigned to the dianions, $\left(\mathrm{C}_{60}\right)_{n}{ }^{2-}$. As another illustration, Figure $1 \mathrm{~b}$ shows an expanded spectrum derived from $\mathrm{C}_{70}$. As well as peaks attributed to $\left(C_{70}\right)_{2}{ }^{-}$, a broad feature at the expected position for $\left(C_{70}\right)_{5}{ }^{2-}$ is seen. Simulations of the isotope structure based on a statistical distribution of ${ }^{12} \mathrm{C}$ and ${ }^{13} \mathrm{C}$ are also presented and are fully consistent with the assignments, thus providing unequivocal confirmation of dianion formation. The smallest observable dianion cluster corresponds to $\mathrm{n}=5$ for both $\mathrm{C}_{60}$ and $\mathrm{C}_{70}$. How can $\left(\mathrm{C}_{60}\right)_{n}{ }^{2-}$ ions form in helium nanodroplets? This question is important because there is no existing mechanism in the literature that accounts for this. Sequential electron attachment can be ruled out because of the strong Coulombic repulsion after addition of the first electron. However, as will be argued shortly, the definitive proof comes from the dependence of the dianion signal on the incoming electron kinetic energy, which is shown in Figure 2. The upper panel includes the yield curve of a selected monoanion, $\left(\mathrm{C}_{60}\right)_{7}{ }^{-}$, together with that of the corresponding dianion, $\left(\mathrm{C}_{60}\right)_{7}{ }^{2-}$. Note that the dianion signal is much weaker than that of the corresponding monoanion, so that the dianion trace in Figure $2 a$ has been expanded to facilitate a comparison. To interpret the fullerene cluster ion yield curves, we first need to consider another ion yield curve, namely that of $\mathrm{He}^{-}$(Figure 2a). $\mathrm{He}^{-}$has been known for some time in the gas phase but has only recently been reported as a product from electron 
impact on helium droplets.[16] Formation of $\mathrm{He}^{-}$requires electronic excitation, as ground-state atomic helium has a negative electron affinity and so cannot attach an electron. $\mathrm{He}^{-}$can be formed from the first excited metastable state of $\mathrm{He}$, the $1 \mathrm{~s} 2 \mathrm{~s} 3 \mathrm{~S} 1$ state, at $19.8 \mathrm{eV}$.[17] In helium droplets, approximately $1.2 \mathrm{eV}$ of additional energy is required for the electron to penetrate the droplet and form a cavity ("bubble") after the inelastic scattering event.[16] Thus at approximately $21 \mathrm{eV}$, the ${ }^{3} \mathrm{~S}_{1}$ state of $\mathrm{He}$, or $\mathrm{He}^{*}$ in shorthand notation, along with a slow electron from the inelastic scattering of the original incoming electron, can form. This slow electron can then attach to $\mathrm{He}^{*}$ to make $\mathrm{He}^{*-}$, which we write as $\mathrm{He}^{-}$from here onwards. However, in helium droplets doped with molecules, the $\mathrm{He}^{*}$ competes with the dopant in attracting the slow electron, and in relatively small droplets, the dopant usually wins because of its higher polarizability than $\mathrm{He}^{*}$ and thus attracts the electron through a stronger charge-induced dipole interaction (plus electrostatic interactions where appropriate). Only when the droplets are sufficiently large ( $10^{5}$ helium atoms[16]), allowing $\mathrm{He}$ to be made at a considerable distance from any dopant, can $\mathrm{He}^{-}$form. $\mathrm{He}^{-}$occupies a bubble because of strong repulsive interactions with neighboring helium atoms.

$\mathrm{He}^{-}$is metastable, with a lifetime depending on the state occupied, but the longest lived is the $1 s 2 s 2 p{ }^{4} P_{5 / 2}$ state with a lifetime of $365 \mathrm{~ms}$ in the gas phase.[18] The short lifetime makes it difficult to study reactive collisions of $\mathrm{He}^{-}$in the gas phase, and no such studies have been reported thus far. However, if the $\mathrm{He}^{-}$bubble travels at the Landau velocity for superfluid ${ }^{4} \mathrm{He}$ (ca. $60 \mathrm{~ms}^{-1}$ ), then it can traverse a droplet of the size employed in the current work in $<1 \mathrm{~ns}$. In fact, the available experimental evidence suggests that the lifetime of $\mathrm{He}^{-}$in helium droplets is much longer than in the gas phase.[16] Thus, once formed, $\mathrm{He}^{-}$will be attracted to a molecular dopant and has ample time to react. The yield curves for the monoanions and dianions selected for illustration in Figure $2 \mathrm{a},\left(\mathrm{C}_{60}\right)_{7}^{-}$ and $\left(\mathrm{C}_{60}\right)_{7}{ }^{2-}$, differ dramatically. On the other hand, the yield curves for $\mathrm{He}^{-}$and $\left(\mathrm{C}_{60}\right)_{7}^{2-}$ are similar and show a relatively narrow resonance peaking near $22 \mathrm{eV}$. Given the overwhelming similarities of their anion yield curves, the $\left(\mathrm{C}_{60}\right)_{n}{ }^{2-}$ anions must be formed by a two-electron electron transfer from $\mathrm{He}^{-}$, a new means of preparing dianions. Figure $2 \mathrm{~b}$ is similar to $2 \mathrm{a}$ but shows data for $\mathrm{C}^{70}$ clusters. Again the similarity between the ion yield curves for $\left(\mathrm{C}_{70}\right)_{7} 7^{2-}$ and $\mathrm{He}^{-}$is striking, showing that the $\left(\mathrm{C}_{70}\right)_{n}{ }^{2-}$ dianions also derive from double electron transfer from $\mathrm{He}^{-}$.

Both the $\mathrm{He}^{-}$and fullerene cluster dianion yield curves are asymmetric and have underlying structure. This is seen most clearly in the $\left(\mathrm{C}_{70}\right)_{n}{ }^{2-}$ curve in Figure $2 \mathrm{~b}$, where a second peak lies approximately $1 \mathrm{eV}$ above the strongest peak: Indeed, the asymmetry continues to higher energies, presumably because other weak and unresolved peaks contribute. These additional peaks are consistent with production of $\mathrm{He}^{-}$in various metastable excited electronic states lying above the lowest observed state (1s2s $\left.2 p{ }^{4} \mathrm{P}\right)$. For example, the $1 \mathrm{~s} 2 \mathrm{p}{ }^{3} \mathrm{P}$ state of neutral He is $1.14 \mathrm{eV}$ above the $1 \mathrm{~s} 2 \mathrm{~s}^{3} \mathrm{~S}$ state,[19] and an electron attached to the former would be consistent with the second peak in both the $\mathrm{He}^{-}$and fullerene dianion yield curves. However, the asymmetry is far more prominent in the case of the dianion curves than in the $\mathrm{He}^{-}$curve, and we attribute this feature to the enhanced reactivity of the more highly electronically excited $\mathrm{He}^{-}$ions. To assess the energetic viability of a twoelectron transfer from $\mathrm{He}^{-}$to a fullerene cluster, we first considered the $\mathrm{C}_{60}$ monomer. The dianion, $\left(C_{60}\right)^{2-}$, has been observed previously in experiments and appears to be metastable with respect to the monoanion and a free electron. $[6,7,20,21]$ The first adiabatic electron affinity of $C_{60}$ has been determined experimentally as $2.6835+/-0.0006 \mathrm{eV}$.[22] The second electron affinity of $\mathrm{C}_{60}$ has not been measured, but a theoretical estimate puts it close to $-0.4 \mathrm{eV},[23]$ and this negative value is consistent with the reported metastability of $\left(\mathrm{C}_{60}\right)^{2-}$. Given that the first ionization energy of $\mathrm{He}$ is $24.56 \mathrm{eV}$ and the electronic excitation energy contained within the lowest state of $\mathrm{He}^{-}$is 
approximately $19.7 \mathrm{eV}$, the energetic deficit is $2.6 \mathrm{eV} .\left(\mathrm{C}_{60}\right)^{2-}$ should therefore not be produced in a low-temperature collision between $\mathrm{C}_{60}$ and the lowest metastable state of $\mathrm{He}^{-}$, which is consistent with our experiments. A similar conclusion can be reached for $\mathrm{C}_{70}$ on the basis of reported electron affinities. [23] The double electron affinities of $\left(C_{60}\right)_{n}$ and $\left(C_{70}\right)_{n}$ clusters should be larger than those of the monomers because a more diffuse excess-electron distribution is possible. The only reported value for the double electron affinity of a $\left(C_{60}\right)_{n}$ cluster is an estimate of $3.86 \mathrm{eV}$ for $\left(\mathrm{C}_{60}\right)_{2}$ from some relatively crude ab initio calculations.[24] Given the lack of electron affinity data for fullerene clusters, we have performed DFT calculations on $\left(C_{60}\right)_{n}$ clusters and their anions for $n=1-5$ as part of this work, and Figure 3 summarizes the findings graphically. The addition of two electrons can create a singlet or triplet dianion, but the electron affinities are not affected significantly by the spin state so this can be ignored. As Figure 3 shows, the second electron affinity for $n \geq 2$ is calculated to be positive, and at around $n=4$, the formation of the dianion becomes thermodynamically allowed in a two electron transfer from $\mathrm{He}^{-}$. The mechanism by which $\mathrm{He}^{-}$transfers its two loosely bound electrons to the dopant remains to be determined. In particular, is it a two-step reduction or a concerted two electron process? A clue can be found in the anion yield curves in Figure 2 . The curves for the $\left(C_{60}\right)_{7}^{-}$and $\left(C_{70}\right)_{7}^{-}$monoanions, which are typical of those of other $\left(C_{60}\right)_{n}^{-}$and $\left(C_{70}\right)_{n}^{-}$ions, are broad and consistent with direct electron attachment as the main source of monoanions. In a sequential two-electron transfer from $\mathrm{He}^{-}$, one might occasionally expect the second step to fail, delivering a peak at approximately $22 \mathrm{eV}$ in the monoanion yield curve matching that of the lowest $\mathrm{He}^{-}$resonance. A small peak is seen at $22 \mathrm{eV}$ for the monoanion signals shown in Figure 2 but the equivalent peak is absent for cluster monoanions with $n>7$. The absence of the $22 \mathrm{eV}$ peak for the monoanions of larger clusters suggests that this peak is the result of some instability of the smaller cluster dianions with respect to Coulomb explosion, leading to monoanions such as $\left(\mathrm{C}_{60}\right)_{7}^{-}$and $\left(\mathrm{C}_{70}\right)_{7}^{-}$. The evidence therefore suggests that dianions derive from a concerted two-electron transfer from $\mathrm{He}^{-}$. A single-step twoelectron transfer also avoids the challenge of overcoming the Coulomb repulsion in the second step of a sequential electron transfer mechanism. A concerted two-electron transfer is consistent with the strong electron correlation expected between the outermost electrons in doubly excited electronic states. In negatively charged doubly excited ions, this effect is accentuated by the excess negative charge, which shields the excited electrons from the nuclear charge and often results in electron correlation providing the majority of the electron binding energy.[25, 26]

However, after the two-electron transfer the cation and dianion must overcome Coulomb attraction to separate. A possibility is that electron transfer occurs at long-range. On the basis of plausible assumptions about the double electron affinities of the fullerene clusters, together with the twoelectron ionization energy of $\mathrm{He}^{-}$(ca. $5 \mathrm{eV}$ ), we can use a simple harpoon model to estimate that two-electron transfer is possible at distances between the $\mathrm{He}^{-}$and the fullerene cluster in excess of $10 \AA$. As the $\mathrm{He}^{+}$is then dragged through the droplet towards the fullerene dianion, $\mathrm{He}_{2}{ }^{+}$can form. As the dissociation energy $\left(D_{e}\right)$ of $\mathrm{He}_{2}{ }^{+}$is near to $2.5 \mathrm{eV}$,[27] the kinetic energy release from forming this cation could allow the cation and dianion to separate.

To summarize, dianions derived from doped helium nanodroplets have been detected for the first time. These anions are formed by a new mechanism, concerted double electron transfer from $\mathrm{He}$, an exotic entity formed in liquid helium at electron impact energies near $22 \mathrm{eV}$. This process is represented by the reaction:

$$
\mathrm{He}^{-}+\left(\mathrm{C}_{60}\right)_{n}+\text { solvent } \rightarrow \mathrm{He}_{2}^{+}+\left(\mathrm{C}_{60}\right)_{n}^{2-}+\text { solvent }
$$


where the solvent here is liquid helium (a video illustration of the mechanism of reaction (1) is provided in the Supporting Information). In this study, $\left(\mathrm{C}_{60}\right)_{n}{ }^{2-}$ and $\left(\mathrm{C}_{70}\right)_{n}{ }^{2-}$ ions were observed but the method used should be transferable to other types of dianions, both in isolation and in combination with other molecules, such as water. More generally this work will open the way for a wider study of the chemistry of $\mathrm{He}^{-}$, a new electron donor.

\section{Acknowledgements}

This work was given financial support by the Austrian Science Fund (FWF), Wien (P23657, P24443, and 1978).

\section{Experimental Section}

Helium nanodroplets were produced by expanding high-purity gaseous helium at a stagnation pressure of 24 bar and a temperature of $9.6 \mathrm{~K}$ through a $5 \mathrm{~mm}$ pinhole into a vacuum. Under these conditions the average number of helium atoms per droplet was in the region of $3 \times 10^{5}$. The expansion was skimmed to form a collimated droplet beam, and this was then passed through a heated pick-up cell containing either $\mathrm{C}_{60}$ or $\mathrm{C}_{70}$ (SES Research, purity $99.95 \%$ and $99 \%$, respectively). After the pick-up of fullerene molecules from the vapor produced in the oven, the droplets passed through a skimmer to enter another differentially pumped chamber, where they were exposed to an electron beam of variable energy $(0-150 \mathrm{eV})$. Any ions produced were then extracted into a highresolution and high-repetition-rate reflectron time-of-flight mass spectrometer (Tofwerk).

\section{Keywords}

density functional calculations · doubly charged anions · electron transfer · fullerenes $\cdot$ helium nanodroplets

\section{References}

[1] M. K. Scheller, R. N. Compton, L. S. Cederbaum, Science 1995, 270, 1160.

[2] A. I. Boldyrev, M. Gutowski, J. Simons, Acc. Chem. Res. 1996, 29, 497.

[3] A. Dreuw, L. S. Cederbaum, Chem. Rev. 2002, 102, 181.

[4] R. C. Dougherty, J. Chem. Phys. 1969, 50, 1896.

[5] S. Schauer, P.Williams, R. N. Compton, Phys. Rev. Lett. 1990, 65, 625. 
[6] R. L. Hettich, R. N. Compton, R. H. Ritchie, Phys. Rev. Lett. 1991, 67, 1242.

[7] P. A. Limbach, L. Schweikhard, K. A. Cowen, M. T. McDermott, A. G. Marshall, J. V. Coe, J. Am. Chem. Soc. 1991, 113, 6795.

[8] A. Herlert, S. Krckberg, L. Schweikhard, M. Vogel, C.Walther, Phys. Scr. 1999, T80, 200.

[9] C. Stoermer, J. Friedrich, M. M. Kappes, Int. J. Mass Spectrom. 2001, 206, 63.

[10] C. Yannouleas, U. Landman, A. Herlert, L. Schweikhard, Phys. Rev. Lett. 2001, 86, 2996.

[11] G. Khairallah, J. B. Peel, Chem. Phys. Lett. 1998, 296, 545.

[12] X. B. Wang, C. F. Ding, L. S. Wang, Phys. Rev. Lett. 1998, 81, 3351.

[13] L. S. Wang, C. F. Ding, X. B. Wang, J. B. Nicholas, Phys. Rev. Lett. 1998, 81, 2667.

[14] S. Denifl, F. Zappa, I. Mhr, J. Lecointre, M. Probst, T. D. Mrk, P. Scheier, Phys. Rev. Lett. 2006, 97, 043201.

[15] F. Zappa, S. Denifl, I. Mhr, A. Bacher, O. Echt, T. D. Mrk, P. Scheier, J. Am. Chem. Soc. 2008, 130, 5573.

[16] A. Mauracher,M. Daxner, J. Postler, S. E. Huber, P. Scheier, J. P. Toennies, J. Phys. Chem. Lett. 2014, 5, 2444.

[17] P. Kristensen, U. V. Pedersen, V. V. Petrunin, T. Anderson, K. T. Chung, Phys. Rev. A 1997, 55, 978.

[18] U. V. Pedersen, M. Hyde, S. P. Møller, T. Anderson, K. T. Chung, Phys. Rev. A 2001, 64, 012503.

[19] A. Kramida, Y. Ralchenko, J. Reader, and NIST ASD Team (2012). NIST Atomic Spectra Database (version 5.0). Available from http://physics.nist.gov/asd [Tuesday, 10-Sep-2013 11:47:31 EDT]. National Institute of Standards and Technology, Gaithersburg, MD.

[20] B. Liu, P. Hvelplund, S. B. Nielsen, S. Tomita, Phys. Rev. Lett. 2004, 92, 168301.

[21] S. Tomita, J. U. Andersen, H. Cederquist, B. Concina, O. Echt, J. S. Forster, K. Hansen, B. A. Huber, P. Hvelplund, J. Jensen, B. Liu, B. Manil, L. Manoury, S. Brønsted Nielsen, J. Rangama, H. T. Schmidt, H. Zettergren, J. Chem. Phys. 2006, 124, 024310.

[22] D.-L. Huang, P. D. Dau, H.-T. Liu, L.-S. Wang, J. Chem. Phys. 2014, 140, 224315.

[23] H. Zettergren, M. Alcam, F. Martn, Phys. Rev. A 2007, 76, 043205.

[24] K. H. Lee, H. M. Lee, W. R. Lee, S. S. Park, H. Lee, T. Y. Park, X. Sun, K. Nasu, Synth. Met. 1997, 86, 2389.

[25] T. Andersen, Phys. Rep. 2004, 394, 157.

[26] D. J. Pegg, Rep. Prog. Phys. 2004, 67, 857.

[27] C.W. Bauschlicher, Jr., H. Partridge, Chem. Phys. Lett. 1989, 160, 183. 


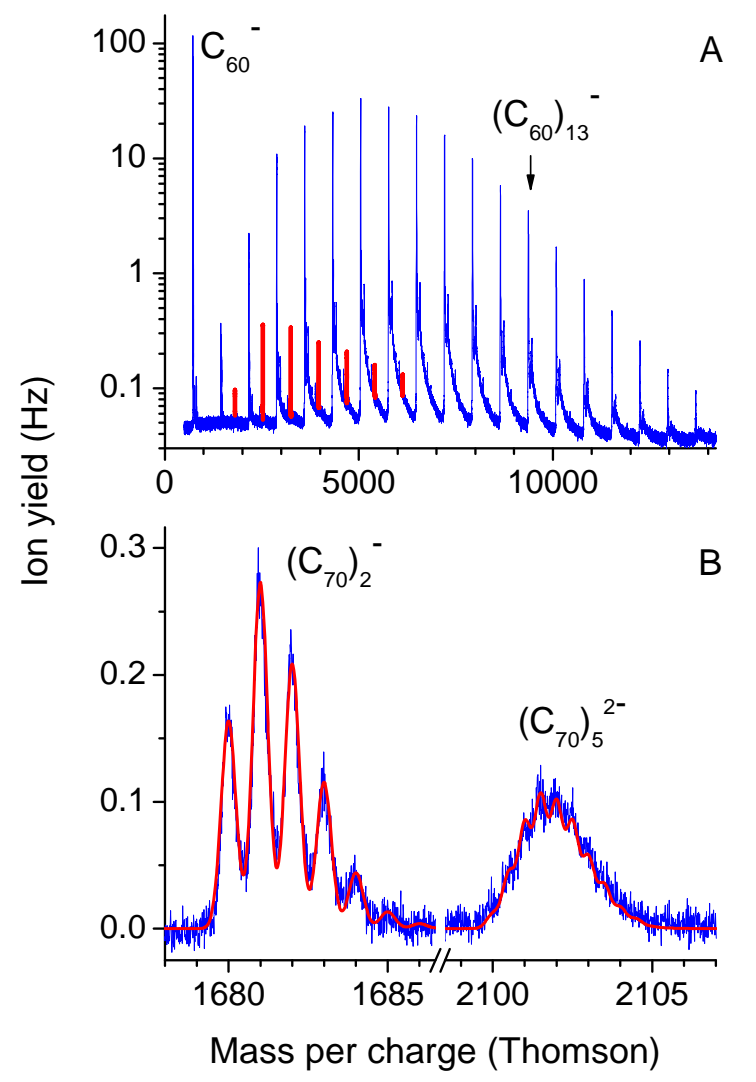

Figure 1: a) Survey mass spectrum showing dominant peaks from $\left(\mathrm{C}_{60}\right)_{n}{ }^{-}$anions. The weaker features marked in red arise from $\left(\mathrm{C}_{60}\right)_{n}{ }^{2-}$ dianions. Note the logarithmic vertical scale. b) An expanded view of the mass spectrum from $C_{70}$ clusters focusing on two specific anions, $\left(C_{70}\right)_{2}{ }^{2-}$ and $\left(C_{70}\right)_{5}{ }^{2-}$. Also shown (in red) is a simulation of the isotope structure based on natural isotope abundances. 


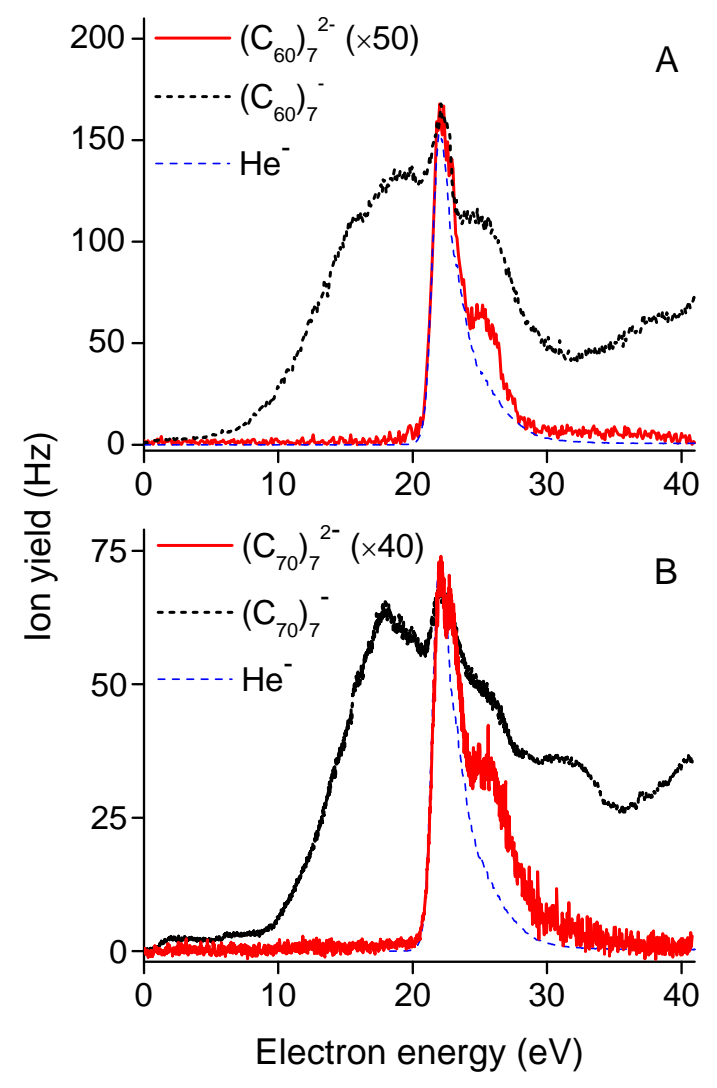

Figure 2a) Comparison of anion yield curves as a function of incident electron kinetic energy for $\mathrm{He}^{-}$, $\left(\mathrm{C}_{60}\right)_{7}^{-}$, and $\left(\mathrm{C}_{60}\right)_{7}{ }^{2-}$. b) Parts of the anion yield curves for $\mathrm{He}^{-},\left(\mathrm{C}_{70}\right)_{7}^{-}$, and $\left(\mathrm{C}_{70}\right)_{7}{ }^{2-}$. The yield curves for both $\mathrm{He}^{-}$and the fullerene cluster dianions are asymmetric, which is attributed to the production of $\mathrm{He}^{-}$in states above the lowest ${ }^{4} \mathrm{P}$ state. The more pronounced asymmetry for the dianion curves suggests that the excited states are more reactive than the lowest state of $\mathrm{He}^{-}$. 


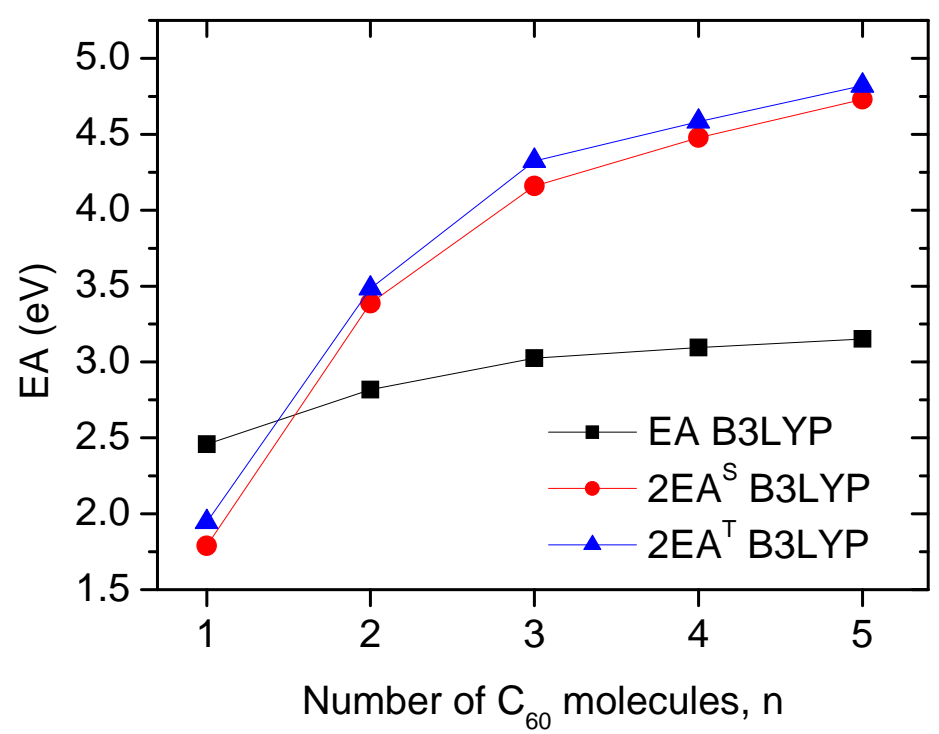

Figure 3. Single and double vertical electron affinities (designated as $E A$ and $2 E A$ ) of $\left(C_{60}\right)_{n}$ clusters as determined from DFT calculations (B3LYP/6-31+G(d)). The calculated electron affinities for both $\mathrm{C}_{60}$ and $\left(C_{60}\right)_{2}$ are insensitive to modest changes in geometry, and so the adiabatic and vertical electron affinities are likely to be comparable. The double electron affinity curves for singlet (2EA ${ }^{S}$ ) and triplet $\left(2 \mathrm{EA}^{\mathrm{T}}\right)$ states of the dianion are also shown and very similar. 